\title{
Design of Fault Diagnosis of Automobile Lights Based on CAN/LIN Bus
}

\author{
You Ren, Wei Sun \\ College of Electrical and Information Engineering, Hunan University, Changsha, 410082, China \\ E-mail: you10906@126.com
}

\begin{abstract}
Based on the research of the CAN/LIN bus communication protocol, an automobile lamps diagnostic system based on the CAN/LIN bus is designed. After the completion of the software and hardware designs of CAN nodes, LIN nodes and CAN/LIN nodes, the faults of automobile lamps can be successfully diagnosed. LIN node collects light switches and the gateway controls the car light's status via detecting the data frame on the LIN bus. The design and complementation of CAN node, LIN node and CAN/LIN gateway was finished, which made it possible to detect failures in vehicle.
\end{abstract}

Keywords: CAN/LAN BUS; Fault Diagnosis; Automobile Lamps

\section{INTRODUCTION}

The Controller Area Network (CAN) which is a serial data communication bus was designed by the Germany Robert Bosch Company in the 1930's to make the real-time data exchange of modern automobiles come true[1]. It was originally designed to solve the problems of the serial communication of passenger cars. But with an increasing number of the electronic equipments used on vehicles, it has been widely used in the electronic systems on vehicles.

Currently, the CAN-based failure diagnostic interface has begun to be used in all the passenger cars in Europe [2]. When there is a fault in automobiles headlights, it is very important to find out the faulty parts and the corresponding causes. Fault diagnosis of automobile lights is part of the vehicle diagnostic system. It can find lights failure, record and send fault code and cut off the power of fault light. Due to the novelty of the technology and the complexity of the diagnostic protocol, the development and application of the vehicle network diagnostic techniques is not yet mature. A simple and practical system of fault diagnosis of automobile headlights was designed in this paper, which uses dedicated automotive electronic chip and high performance microcontroller and can accurately determine the failure of the lights and the type of the fault.

\section{SySTEM NETWORK DESIGN}

A hybrid network system which is made up of a CAN network and a LIN network and which makes these two networks' information fused by means of gateways is designed in this paper. All the nodes are independent only when the data are exchanged do the nodes communicate through a bus. CAN network is made up of the front of the body control module ( FBCM ), the rare of the body control module (RBCM) and a PC node and the front of the bus control module is the hardcore of the whole system. FBCM not only works as a function controller of the automobile body system but also works as a CAN/LIN gateway which can process the information of the whole network and the RBCM node can drives the rare lamps by means of receiving the information sent from the CAN network. LIN network is made up of the FBCM node and the MLS (Main Light Switch) node with FBCM working as the main node to manage the LIN network through a scheduling list and MLS being used to collect the information of the combined switch and to send information about switch situation to the gateway. Gateway is the core of communication. It can make the information on these two networks shared and make the network self-manage and make faults be diagnosed. Figure.1 shows the system's

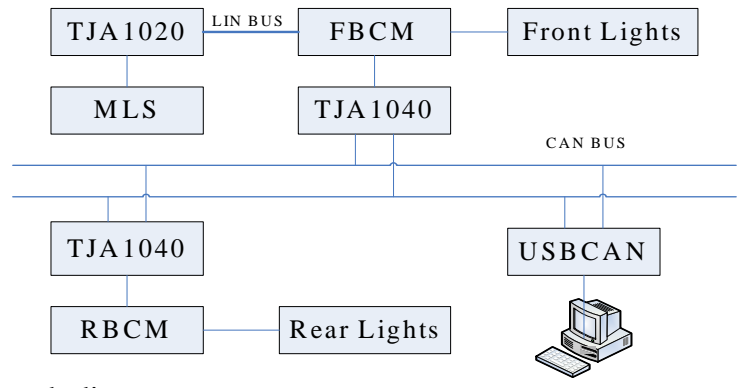

network diagram.

Figure 1. System's network diagram

\section{The DESIgN OF THE NETWORK NODE}

Master node is made up of a control chip, a system basic chip MC33742, CAN receiver TJA1040 and a LIN receiver TJA1020. Its master node is Freescale Company's 16 micro controller MC9S12XET256MAL single-chips. It possesses a strong communication ability because this micro controller is equipped with the CAN control module and therefore there is no need to design another micro controller to connect with the CAN controller's circuit, which improves the stability of this system [3]. In this system, it can not only work as a CAN node to control automobile headlamps, what is more important is that it can work as a gateway to link CAN network with LIN network. The MC33742 is SPIcontrolled System Basis Chips (SBCs) combining many frequently used functions along with a CAN2.0-compliant transceiver [4]. CAN high-speed physical bus interface with TXD and RXD fault diagnostic capability and enhanced 
protection features and possesses battery management function. Take FBCM node as an example, hardware frame diagram is as followed.

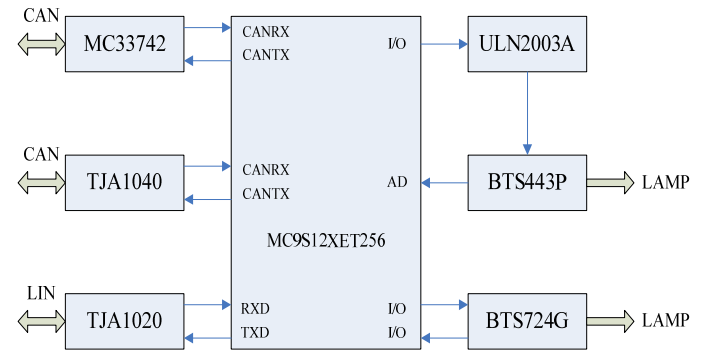

Figure 2. Hardware frame diagram

Upper computer software uses the USBCAN switch to act as a CAN node to link the CAN network. USBCAN-2A is an intelligent PC-CAN interface module which is compatible with USB1.1 and supports one or two CAN channels. Each channel integrates independent electrical isolation circuit. And with the module and USB port, a control node of CAN-bus can be formed by connecting the $\mathrm{PC}$ to the CAN network. This node is used to read real-time fault code in FBCM and RBCM nodes to confirm faulty parts and their fault types.

LIN node is made up of Freescal 16 single chip which acts as micro controller and LIN Enhanced Physical Interface 33661. The 33661 is a Physical Layer component dedicated to automotive LIN sub-bus applications. In this system, LIN node is mainly used to collect information about the combined switch and sends the information about the light switch to the gateway in the form of LIN data frame. After having analyzed LIN frames, the gateway would send the LIN frames to the CAN network transparently and then drives different automobile headlamps to perform corresponding actions.

IV. Fault diagnosis of Automobile Lights Based ON CAN/LIN BUS

The fault diagnosis system is applied by applying the vehicle diagnosis system on the communication system. This paper focuses on the detection and diagnosis of the link faults. The link fault is mainly resulted from the short circuit

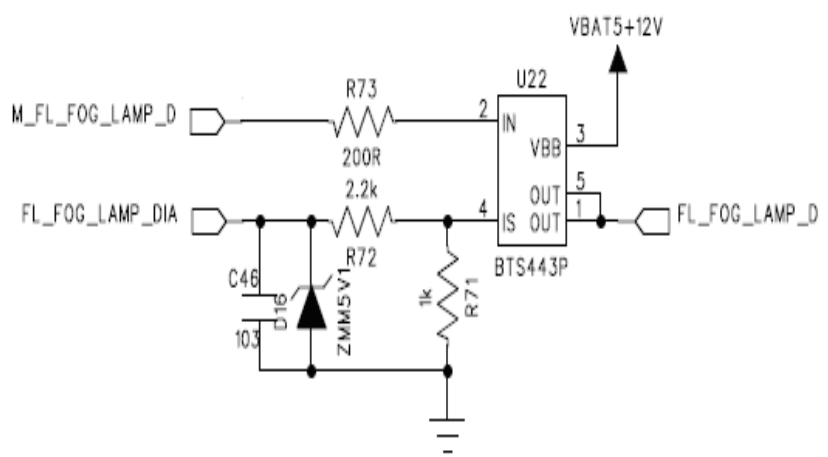

Figure 3. BTS443p Drive Circuit and the open circuit of communication lines. Using intelligent drive chips will bring considerable convenience to the diagnosis. This kind of chip has both the driving function and the diagnostic function. Therefore, when the actuator is driven, an information is fed back to detect the actuator. This design is by detecting the feedback information of driving chip to judge the state of lights.

Various kinds of intelligent driving chips are adopted to implement the diagnostic function. The BTS443P and BTS724G are adapted to work as the driving chip of automobile headlamps. All of them possess the diagnostic function, short circuit protection function and overload protection function. Because of their different driving powers and different fault diagnosis principles, they are used to drive different headlights. BTS742G is used to drive lowpower headlamps and to detect their faults and to diagnose and to reflect digital inputs. The main controller can read levels through the $\mathrm{I} / \mathrm{O}$ interface and can diagnose the fault according to the logical relations of the truth tables. It is shown as Figure 7. BTS443P is mainly used to drive the headlamps with relatively high power and can diagnose and reflect the analog inputs. It is shown as Figure 3. The main controller can read the feedback values through the $\mathrm{A} / \mathrm{D}$ interface and thus find out the fault causes. But when the feedback values are not within a certain range, the fault codes of the corresponding parts will be kept in the EEPROM, waiting for the detection of the upper computers. Take FBCM node for example. High beams, light lamps and fog lamps have high power. Therefore, BTS443P is adopted to drive them and as for turn signals, position lamps and brake lights which have relatively low power, BTS724G is used to drive them. TABLE I shows the BTS443p's normal sample values.

TABLE I. BTS443P'S NORMAL SAMPLE VALUES

\begin{tabular}{|c|c|c|c|}
\hline LAMP & $\begin{array}{c}\text { normal } \\
\text { sample } \\
\text { values }\end{array}$ & LAMP & $\begin{array}{c}\text { normal } \\
\text { sample } \\
\text { values }\end{array}$ \\
\hline $\begin{array}{c}\text { Right High } \\
\text { Beams }\end{array}$ & $5800 \sim 6200$ & $\begin{array}{c}\text { Former Right Fog } \\
\text { Lights }\end{array}$ & $4400 \sim 4800$ \\
\hline $\begin{array}{c}\text { Left High } \\
\text { Beams }\end{array}$ & $5950 \sim 6350$ & $\begin{array}{c}\text { Former Left Fog } \\
\text { Lights }\end{array}$ & $4650 \sim 5050$ \\
\hline $\begin{array}{c}\text { Right Near } \\
\text { Lights }\end{array}$ & $4500 \sim 4850$ & $\begin{array}{c}\text { Rear Right Fog } \\
\text { Lights }\end{array}$ & $2300 \sim 2800$ \\
\hline $\begin{array}{c}\text { Left Near } \\
\text { Lights }\end{array}$ & $4450 \sim 4900$ & $\begin{array}{c}\text { Rear Left Fog } \\
\text { Lights }\end{array}$ & $2350 \sim 2800$ \\
\hline
\end{tabular}

When the sampling value is greater than the normal sampling values, the short circuit may occur in the corresponding lamps. But when the sampling value is less than the normal sampling value, short circuit may happen. Therefore, by detecting the BTS443P's pin signals, it can tell if faults occur.

Through the study of the ISO14229 protocol, the means to detect faults are designed. The master chip collects realtime feedbacks of driver chips and that faults occur or not or which kind of faults happens can be confirmed according to the feedbacks. Take a right high beam for example, when the AD sampling value is less than 5800 , open circuit may 
happen according to the BTS443P truth value. But when the $\mathrm{AD}$ sampling value is more than 5800 , some other causes may lead to it. In order to avoid false diagnoses, the ISO14229 protocol is adopted to confirm the faults. Principle: a test is taken every 20ms and 50 continuous tests are taken as a circulation. When a fault is confirmed in a circulation, the corresponding fault codes will be written in the EEPROM. Different codes will drive corresponding lamps according to the messages sent by LIN code. Therefore, fault codes will be kept in the corresponding EEPROM and the upper computer will read fault codes according to the protocol. After the fault has been confirmed, frame information will be sent by FBCM and RBCM, asking the node of upper computer to read fault codes and the node find out the faulty parts and their faults according to fault codes. The flow chart is as shown in Figure 5. Where Diagnostic Trouble Code (DTC) is defined as shown in TABLE II.

When a fault is confirmed by the FBCM and RBCM node, a fault message will be sent to the node of upper computer. After having received the message, the upper computer will send frame news asking the nodes of FBCM and RBCM to read the fault codes which are kept in the EEPROM and to send the fault codes to the node of upper

TABLE II. Diagnostic TROUBle CodE STATUS BIT

\begin{tabular}{|c|c|c|}
\hline Bit & Bit Name & Description \\
\hline $\mathbf{0}$ & Test_Failed & $\begin{array}{l}\text { The result of the most recently } \\
\text { performed test }\end{array}$ \\
\hline 1 & $\begin{array}{l}\text { testFailedThisOperation } \\
\text { Cycle }\end{array}$ & $\begin{array}{l}\text { ' } 0 \text { '=DTC test has not failed this } \\
\text { operation cycle } \\
\text { ' } 1 \text { '=DTC test failed this operation } \\
\text { cycle }\end{array}$ \\
\hline 2 & $\begin{array}{l}\text { testFailedLastOperation } \\
\text { Cycle }\end{array}$ & $\begin{array}{l}\text { '0'=DTC test has not failed last } \\
\text { operation cycle } \\
\text { ' } 1 \text { '=DTC test failed last operation } \\
\text { cycle }\end{array}$ \\
\hline 3 & ConfirmedDTC & $\begin{array}{l}' 0 \text { ' }=\text { Not failure } \\
' 1 \text { '=Confirm failure }\end{array}$ \\
\hline 4 & ClearDTCFlag & Clear the DTC flag \\
\hline 5 & Fault_Counter & Count the number of fault \\
\hline 6 & $\begin{array}{l}\text { testNotCompletedThis } \\
\text { OperationCyce }\end{array}$ & Undefined \\
\hline 7 & $\begin{array}{l}\text { warningIndicatorReques } \\
\text { ted }\end{array}$ & Undefined \\
\hline
\end{tabular}

computer. PC node can be quickly and accurately read to get fault code was to determine the failure of lights. It is shown as Figure4. Because at most 8bytes can be sent in a frame, after having receiving fault codes, the upper computer confirms the faulty parts and the type of fault according to the fault codes and then sends instructions to the nodes to clear fault codes and then do another detection.

\begin{tabular}{|c|c|c|c|c|c|}
\hline \multicolumn{4}{|c|}{ Fit Testing Software of Body Network } & 口回 & $\Sigma 3$ \\
\hline \multicolumn{6}{|c|}{ CAN Node Setting Online Test Fault Diagnosis } \\
\hline \multicolumn{6}{|l|}{ Fault Diagnosis } \\
\hline DETECT DTC & \multicolumn{4}{|c|}{ Fault Code List } & \\
\hline \multirow[b]{2}{*}{ READ DTC } & & Fault Code & \multicolumn{2}{|l|}{ Lights } & $\Delta$ \\
\hline & & 9001 & \multicolumn{2}{|c|}{ Former Right Fog Lights } & \\
\hline \multirow[b]{2}{*}{ CLEAR DTC } & & 9002 & \multicolumn{2}{|c|}{ Former Left Fog Lights } & \\
\hline & $\triangle$ & 9003 & \multicolumn{2}{|c|}{ Rear Right Fog Lights } & \\
\hline \multirow{2}{*}{$\begin{array}{l}\text { Fault Code: } \\
90039005\end{array}$} & & 9004 & \multicolumn{2}{|c|}{ Rear Left Fog Lights } & \\
\hline & $\triangle$ & 9005 & \multicolumn{2}{|l|}{ Right High Beams } & \\
\hline CLOSE & & 9006 & \multicolumn{2}{|l|}{ Right Near Lights } & -1 \\
\hline
\end{tabular}

Figure 4. Fault diagnosis result

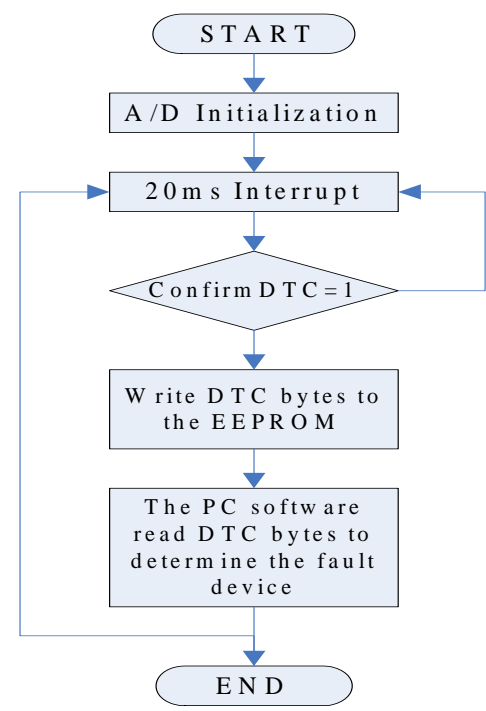

Figure 5. Fault diagnosis result

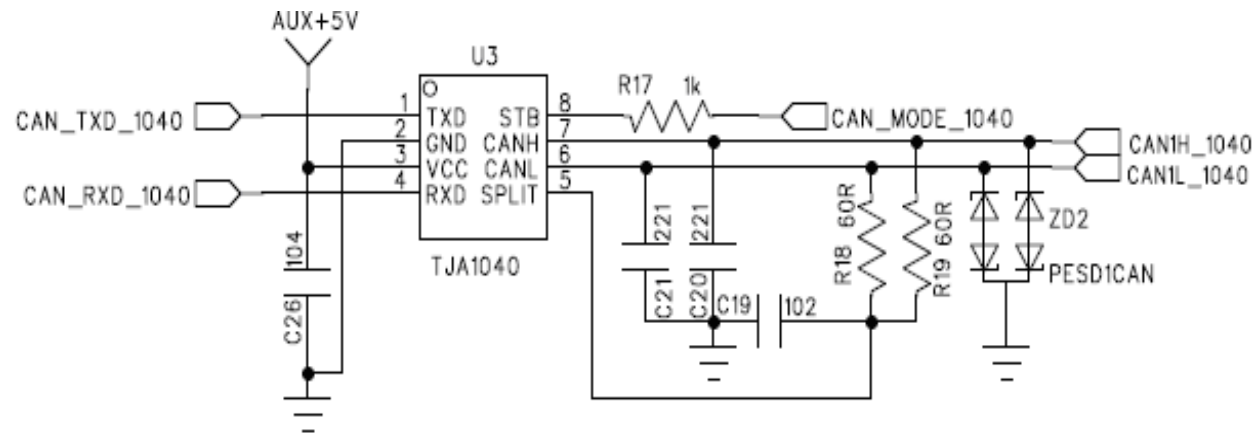

Figure 6. Connection circuit between CAN Bus and CAN Node 


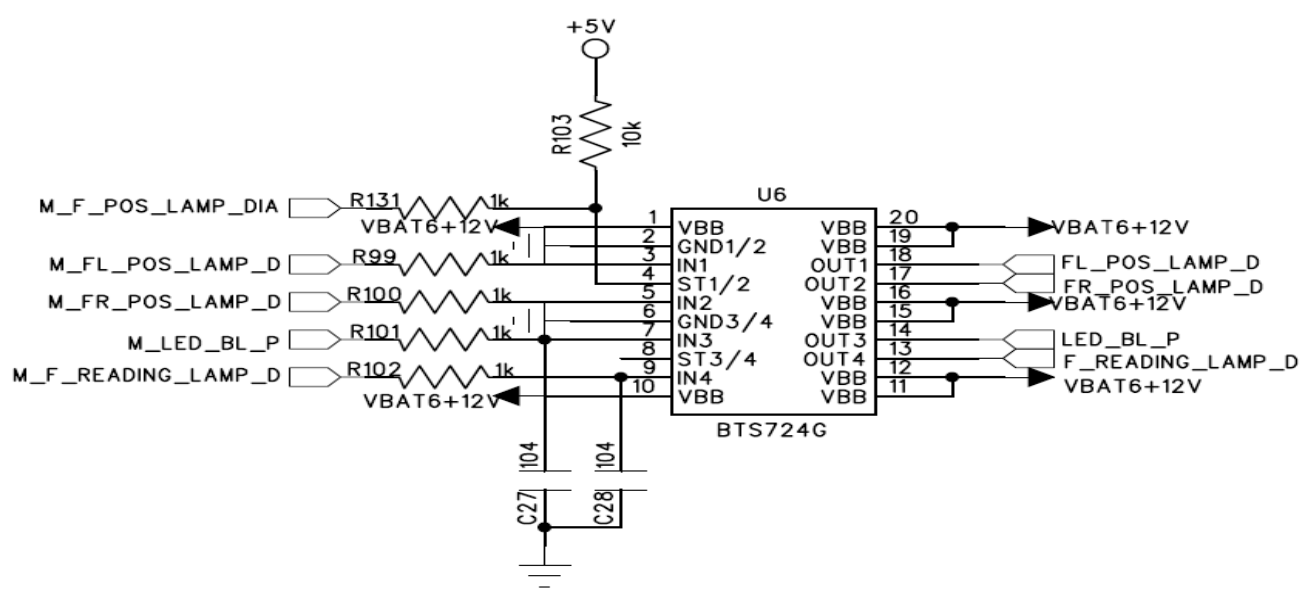

Figure 7. BTS724G Drive Circuit

\section{CONCLUSIONS}

Bases on the research of CAN/LIN communication protocol, a simple but useful automobile lamp diagnostic system is designed. The design of the network layer confirms the ISO15765-2 regulation. The diagnostic system has been applied to a CAN network control system. Its application proves its stability in data transmission and its correctness and real time in fault diagnoses.

\section{RFEFERENCES}

[1] Luo Feng, Sun Zechang, Principle, Design and Application of Automotive CAN Bus System, Publishing House of Electronics Industry, 2010
[2] Li Rui, Wang Jingying, Yao Yan, Design of Vehicular CAN Network Diagnosis Based on ISO15765 , Computer Engineering, 2012, 2, 35-39.

[3] MC9S12XEP100RMV1, REV.1.19, Freescale semiconductor, 2008.

[4] MC33742 Rev 11, Freescale Semiconductor, 2008.

[5] Wu Kuanming, Application System Design and Bus Theory of CAN, Beihang University Publishing Company, 1999

[6] SAE JI939/21 -CAN Data Link Layer -Issue 1994-07, Revised 199807.

[7] Yang Xianhui, Technique and Application of Field Bus, Tsinghua University Publishing Complany, 1999.

[8] Fu Liang, Li Wei. The Development and Design on CAN-bus Controller SJAIOOO. Modem Transportation Technology, 2007,(4). 